\title{
DATA MANAGEMENT BASED ON GEOCODING INDEX AND ADAPTIVE VISUALIZATION FOR AIRBORNE LIDAR
}

\author{
Xiaodong $\mathrm{Zhi}^{\mathrm{a}}{ }^{*}$, Keshu Zhang ${ }^{\mathrm{b}}$, Guozhong $\mathrm{Su}^{\mathrm{b}}$ \\ a School of Remote Sensing Information Engineering, Wuhan University, 129 Luoyu Road, Wuhan 430079, China; \\ ${ }^{\mathrm{b}}$ Academy of opto-electronics, Chinese Academy of Sciences, Beijing 100080, China;
}

KEY WORDS: Laser scanning (LiDAR), Data management, Aerial Survey, Coding, Process modeling

\begin{abstract}
:
With more surveying practice and deeper application, data post-process for airborne LiDAR system has been extracted lots of attention in data accuracy, post-process, fusion, modeling, automation and visualization. However, post-process and flexible visualization were found to be the bottle-neck which limits the LiDAR data usage for industrial applications. The cause of above bottle-neck problems is great capacity for LiDAR system. Thus in article a geocoding index based multivariate data management and adaptive visualization will be studied for based on the feature of airborne LiDAR's data to improve automatization of postprocess and surveying efficiency.
\end{abstract}

\section{INTRODUCTION}

\subsection{Development of LiDAR}

LiDAR (Light Detection and Ranging) is an active detection system of radar, which includes some techniques of laser, Global Position System (GPS) and Inertial Navigation System (INS). It can be used to acquire ground information and stereo information of ground objects through the whole day. LiDAR is the most essential techniques of information acquiring and processing for earth observation system (EOS) plan, with image spectrometer and synthetic aperture radar. In recent decades, air-borne LiDAR technique has been well accepted by developed countries, as an accurate and rapid method of acquiring surface three dimensional information. It has been developed and applied in terrain mapping, environment monitoring, digital city modeling and military applications. This technique only has been developed in several western countries, in aspects of researching in polar region and Mars detection.

Compared to the fast development of the hardware of LiDAR, its data management and handling technologies lag behind comparatively. At present, most of the LiDAR data managing software are provided by some specific companies(like REALM,Optech Co.). These softwares' algorithms are covered secretly which can't be parsed by the users so that thay cannot manipulate and manage those softwares. Besides, there are some standalone universal softwares that are independent to hardware(like LID-MAS,US and TerraSolid, Finland).Those softwares' data management is based on sparse file structures, and theirs algorithms are designed according to the calculations on every single files which are incapable of dealing with large number of data like LiDAR.

\subsection{Characters of LiDAR data}

Datum acquired from Airborne LiDAR, strictly speaking, includes position, attitude(angle), range, time, intensity in flight. The characters of this datum are: a) LiDAR's data is a series of three-dimensional points distributing in the surface of the object.

b) LiDAR's data is in the form of a discrete data distribution, and irregular.

c) LiDAR's data contain too many redundant data, and the density of points is not uneven.

d) The most important character of LiDAR's data is too large in size and content. This led directly to the data organization and management inconveniently, and some existing algorithms are not suitable for airborne LiDAR.

\subsection{Example}

Take AOE-LiDAR system as an example, it can generates up to $10 \mathrm{~Gb}$ laser data and $15 \mathrm{~Gb}$ image data from external digital aerial camera per hour in one platform. Moreover, for resolving the LiDAR data in post-process stage, large amount of intermedia data files are created. Even further, for tuning the data quality, use different parsing parameter for different application purpose. This article will investigate complexity and the way to management these complexity by using geocoding index based multivariate data management and adaptive visualization in post-process stage.

\section{AIRBORNE LIDAR'S DATA ACQUISITION, PROCESS MECHANISM AND DATA TYPES}

\subsection{The mechanism of airborne LiDAR's data acquisition}

The system of airborne LiDAR includes laser scanning, position/ attitude control system and control host. Laser scanning launch and accept laser signals. Position/ attitude system composed by IMU (inertial measurement unit) and DGPS (differential GPS), which can output the position and attitude parameters for airborne platform. Its principle is shown in Fig below:

\footnotetext{
* Xiaodong Zhi. tuladingx@163.com, phone: 0086-1590 7718999
} 


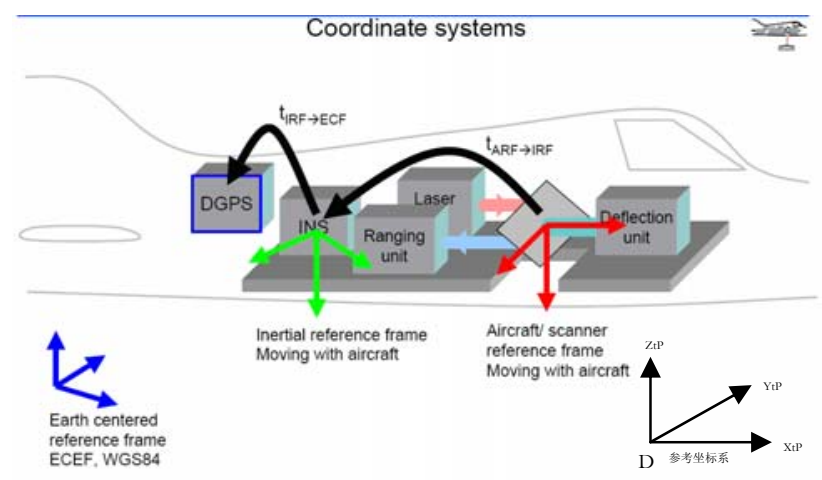

Through the above model, we get the ground 3D point clouds following formula:

$$
\left[\begin{array}{c}
x \\
y \\
z
\end{array}\right]_{W G S-84}=\left[\begin{array}{l}
X_{0} \\
Y_{0} \\
Z_{0}
\end{array}\right]_{W G S-84}+R_{W G S-84(p, o, k)} R_{\operatorname{Re} f(I M U)}\left[\begin{array}{l}
X_{L} \\
Y_{L} \\
Z_{L}
\end{array}\right]_{\text {Laser }}
$$

Ref is the reference coordinate system for IMU, its relationship between IMU coordinate system is restrictionist for resettlement of IMU. So if we set up the conversion relations in POS control system, the output will be in reference coordinate system for IMU.

$\left[X_{L}, Y_{L}, Z_{L}\right]$ is coordinates of point clouds in laser system, and its value is calibrated.

\subsection{The mechanism of airborne LiDAR's data process}

The process of airborne LiDAR is shown in Fig below:

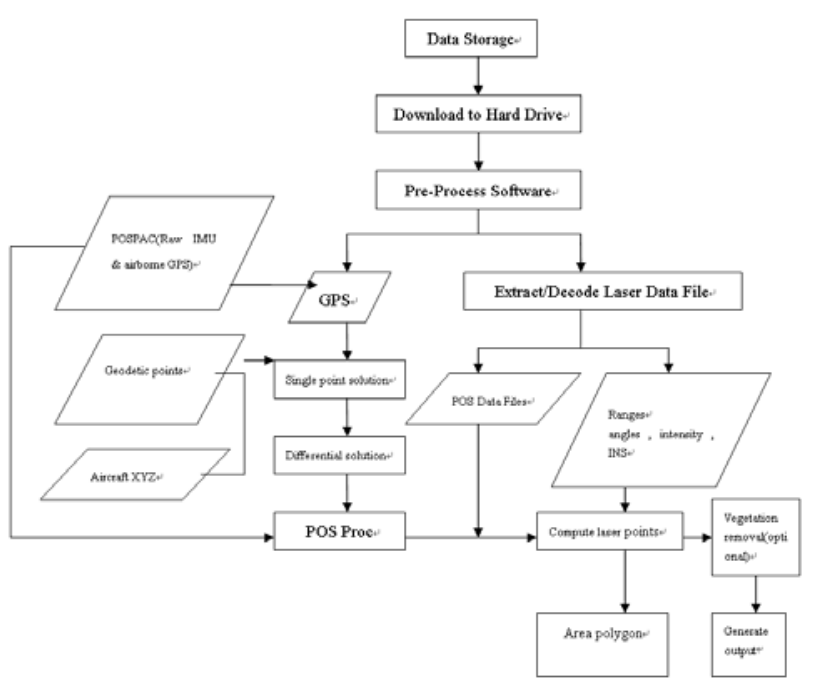

1. Decoding, extract the raw scanning data according to data types, but in data file of any type ,the time sequence is same as raw data. Such as remote station GPS, INS, laser scanning data(range, angle, intensity).

2. Using GPS processing software to resolve the master station and remote station GPS data.

3. Using POS processing software to combine GPS and IMU with smoothing algorithm.

4. Matching multiple data to compute coordinates, in this stage, the main operation is interpolation by time.
5. Computing coordinates of points, it contains correcting, coordinates rotation and computing XYZ values.

6. Outputting data, generating the recognized format file for point clouds.

\subsection{Data types of Airborne LiDAR}

In accordance with its data Solution to classify the role of all kinds of data. The data type of airborne LiDAR contains:

a) Raw Scanning Data

Raw scanning data;

Navigation sbet file from POS processing software;

Survey area data;

b) Middle Processing Data

Laser scanning data (range, angle, intensity);

Time synchronization data;

Equipment status and environment parameters data; Log data in flight;

c) Outputting data

ASCII format XYZI file(X, Y, Z, Intensity);

The internationally popular laser point cloud data files LAS, developed by the ASPRS.

\section{MANAGE POINT CLOUDS}

One LiDAR (laser radar) sensor generates over a hundred millions of XYZ coordinate parameters. These great amount of sample points are described "non-organized". When those LiDAR points are re-built, they become "Cloud". How to organize these non-organised points?

\subsection{Problems}

Those hundreds million points course calculation ball-up, because it is impossible to store all the data into the memories of the most complicated computer. Therefore, the data should be the hard disks which are in larger volumes but slower speed. Handling the large number of data within the hard disks and RAM instead of within computers has some disadvantages in the manipulation. Thus, most practical algorithms use section algorithm to rupture the cloud point into a series of nonoverlaid second level cloud which includes a few sample points. In every section, the data interpolation is independent. Based on this principal, many based on section methods are developed, including the simple rupture and ruptures based on Voronoi line-drawing figuring method. One algorithm to match the data exchange can minimize the time period of visiting hard disk which also compress the time of running. People are still searching for algorithms that can deduce the time of data exchange.

\subsection{Geocoding Index}

Common characteristics of all point clouds are geodetic coordinates. So we should not organize data through files, using geodetic location of every point to manage all data. In this case, for every file below information is must known:

a) In the XY plane the smallest external rectangular for the data in one file;

b) In LiDAR system every scan line's tail data offsets to file starting point;

c) The storage path of every file, this path also can in LAN. 
For above information, statistics them before processing and save this index data to one XML file. Using this index data, we can seamlessly management and view a large number of point clouds. In past, it's difficulty to realize data distribution, because the size of data is too large and beyond the computer. But using geocoding index, we can draw the smallest external rectangular for every file to realize data distribution. And this method is simplicity and intuitive to global view as below Fig shown:

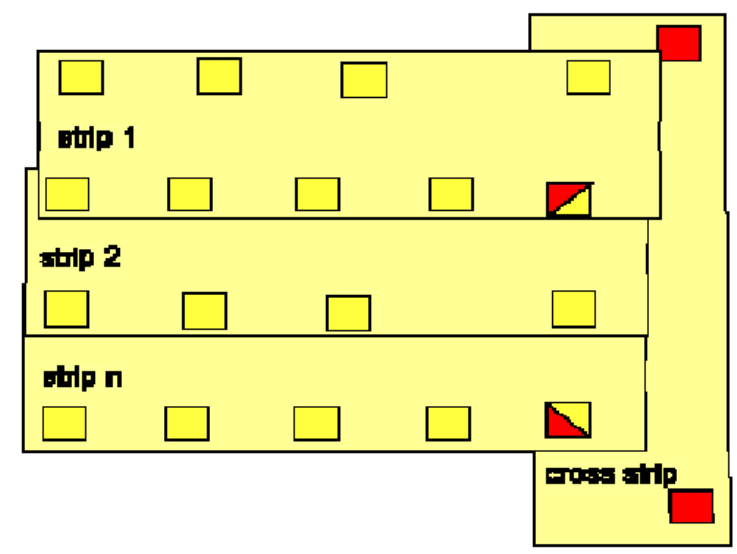

In other hand, in point clouds, scan angle is ignored usually. Scan angle equivalent to scan line for data. And in practice, many data organizations using scan line as the smallest unit. Because the file position of data in one scan line is known, it is not necessary to load data by files. Through select a geodetic area to load dataset which from many files and part of some files, the loading formula is:

In rapid viewing large number of data, simplifying the data can be separated by a number of scanning lines directly reading data. This seamless point cloud data management methods significantly improve the efficiency of the man-machine cooperation.

\subsection{Point cloud pyramid}

The information from human eyes, are obtained based on multiresolution, which also accords with the human cognitive things by coarse-to-fine features, and has used in image processing. LiDAR data is also on a description of the objective world, we can still use the point cloud pyramid organizational mechanisms, and its algorithm is as follows:

Every point cloud file relates to a point cloud index files. This index file contains $n$ levels, as below (4 layers):

\begin{tabular}{|c|}
\hline $3 \times 3$ \\
\hline $9 \times 9$ \\
\hline $27 \times 27$ \\
\hline $81 \times 81$ \\
\hline
\end{tabular}

Every level of index files stored in the information below, taking $3 * 3$ as example:

Recording the coordinate scope for every block;

Recording the offset position in file for this block data.

In LOD algorithm, far from eye point, using $3 * 3$ index; close from eye point, using $81 * 81$ index.

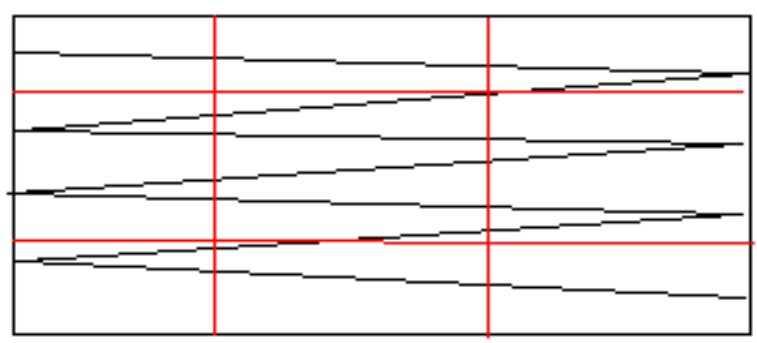

\section{ADAPTIVE VISUALIZATION FOR AIRBORNE LIDAR}

In resolving session of airborne LiDAR, data visualization is usually used in bore-sight calibration, LiDAR's data classification, generating DSM (Digital Surface Model), object reconstruction by fusing LiDAR Data with Photogrammetry, and so on. But in these sessions, not all operation need display full data. For instance, in bore-sight calibration, we only need profile of LiDAR's data to feedback the setting of computing parameters. So a new adaptive visualization algorithm is proposed to improve customer experience and quicken data resolving. In bore-sight calibration:

\subsection{Pitch}

Choose to fly through the cuspate roof back and forth to analyze the airline data. Draw a block that contains the area of the root top among the generated point cloud data, and build up two surfaces. Draw a profile plumbing the roof top in both of the two surfaces to display the profile of the two surfaces together. Measure the errors in the horizontal orientation of the two profiles, and then divide the flying height to come up with a validation value. See the theory in the diagram.

Re-input the value into the LiDAR pre-handling software to regenerate point cloud data. Use the same way to re-build profile. If those two profiles match well, the validation value for Pitching is proved to be valid. If not, go back and redo these steps to revise based on the previous validation value until the two profiles match completely. According to the steps above, not all the profiles data are used in the revising process.

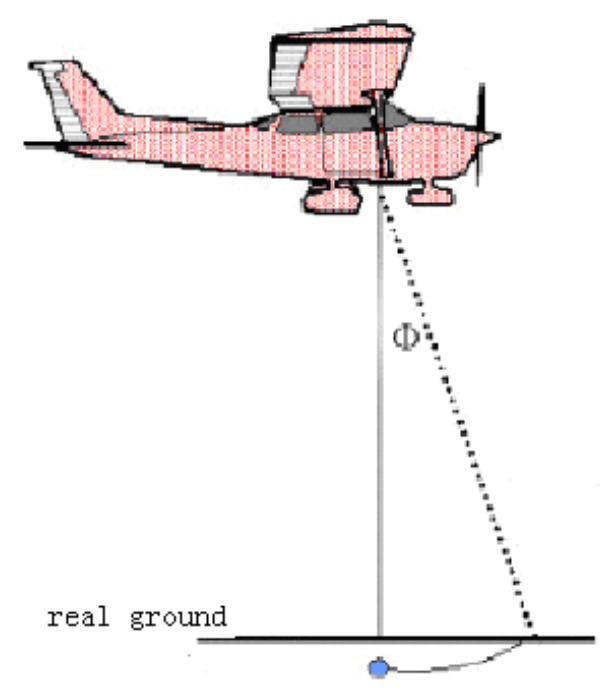

recording point 


\subsection{Roll}

Choose to fly through the path vertically back and forth to analyze the airline data. Draw a surface that contains the area among the generated point cloud data respectively, and build up two surfaces. Measure the errors in the vertical orientation of the two profiles, and then divide the scanning width of LIDAR to come up with a validation value. See the theory in the diagram.

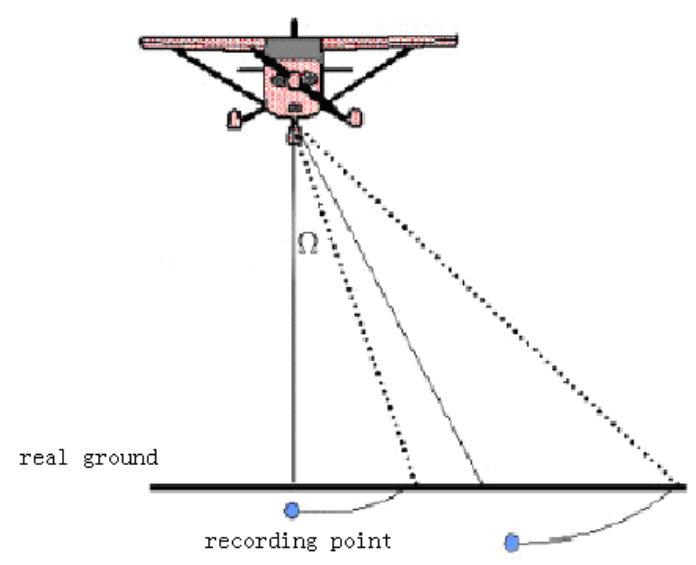

Re-input the value into the LiDAR pre-handling software to regenerate point cloud data. Use the same way to re-build profiles. If those two profiles match well, the validation value for Pitching is proved to be valid. If not, go back and redo these steps to revise based on the previous validation value until the two profiles match completely. According to the steps above, not all the profiles data are used in the revising process.

\subsection{Heading}

Choose to two parallel fly airlines in the same direction to analyze the airline data and Pitch angle. Draw a block that contains the area of the root top among the generated point cloud data, and build up two surfaces. Draw a parallel line across the roof top as profiles. Measure the errors in the horizontal orientation of the two profiles, and then divide the extension of two airlines to come up with a heading validation value. See the theory in the diagram.

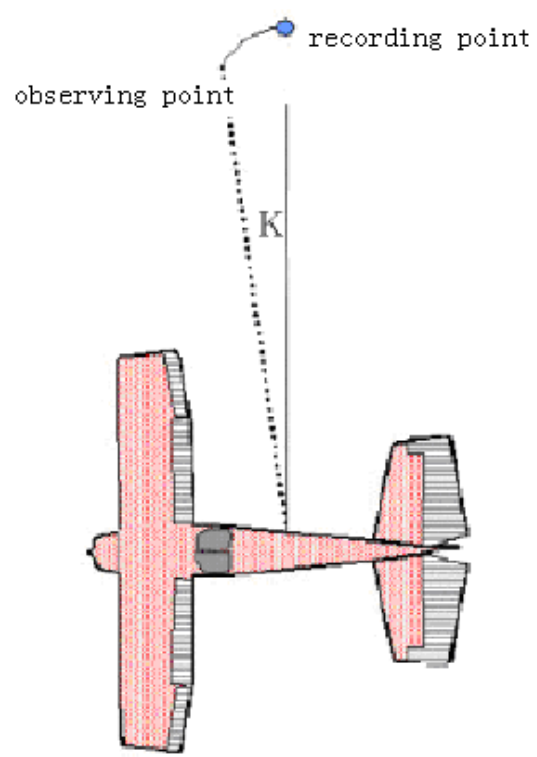

Re-input the value into the LiDAR pre-handling software to regenerate point cloud data. Use the same way to re-build profiles. If those two profiles match well, the validation value for Pitching is proved to be valid. If not, go back and redo these steps to revise based on the previous validation value until the two profiles match completely. According to the steps above, not all the profiles data are used in the revising process.

\section{CONCLUSION}

In conclusion, it is not better for more point clouds to view a large number of data, but according to the purpose of the adjustment from the show adaptation strategies, such manmachine interaction can provide speed, but also be very good visual effect.

\section{REFERENCES}

1. D. M. Barber, J. P. Mills and P. G. Bryan. MAINTAINING MOMENTUM IN TERRESTRIAL LASER SCANNING: A UK CASE STUDY. School of Civil Engineering and Geosciences, University of Newcastle upon Tyne, UK.

2. Dr.-Ing. Rolf Katzenbeisser. ABOUT THE CALIBRATION OF LIDAR SENSORS. TopoSys GmbH D-88214 Ravensburg

3. R. A. Haugerud and D. J. Harding. SOME ALGORITHMS FOR VIRTUAL DEFORESTATION (VDF) OF LIDAR TOPOGRAPHIC SURVEY DATA. U.S. Geological Survey c/o University of Washington.

4. Jungho Im. Object-based Land Cover Classification Using High Posting Density Lidar Data. Environmental Resources and Forest Engineering, State University of New York, College of Environmental Sciences and Forestry.

5. E.P. Baltsavias. Airborne laser scanning: basic relations and formulas. Institute of Geodesy and Photogrammetry, ETHHoenggerberg, CH-8093 Zurich, Switzerland.

6. Xiaohong Zhang. Precise Point Positioning Evaluation and Airborne Lidar Calibration. Danish National Space Center. 